\title{
Frequency metrology by use of quantum interference
}

\author{
N. Ph. Georgiades, E. S. Polzik, ${ }^{*}$ and H. J. Kimble \\ Norman Bridge Laboratory of Physics, 12-33 California Institute of Technology, Pasadena, California 91125
}

Received May 31, 1996

Quantum interference in the rate of two-photon excitation of the $6 S_{1 / 2} \rightarrow 6 P_{3 / 2} \rightarrow 6 D_{5 / 2}$ transition in atomic cesium is exploited to demonstrate phase-sensitive frequency demodulation for an optical interval of $\pm 12.5 \mathrm{THz}$. By thus using atoms as ultrafast nonlinear mixing elements, we suggest and analyze a new avenue for absolute comparisons of a dense set of frequencies over the range of 200-2000 nm. ( 1996 Optical Society of America

Quantum interference (QI) of multiple excitation pathways is ubiquitous in atomic, molecular, and optical physics, with applications to the coherent control of products in a chemical reaction, ${ }^{1}$ phase-sensitive yields in above-threshold ionization, ${ }^{2}$ and the synthesis of atomic wave functions ${ }^{3}$ being but three recent examples. In this Letter we present observations of QI for two-photon excitation whereby interfering paths lead to modulation of excited-state population as a function of the relative phase of three excitation beams with widely separated frequencies (corresponding to 852, 884 , and $917 \mathrm{~nm}$ ). By using an atom as an ultrafast nonlinear mixing element, we demonstrate optical demodulation for an interval of $\pm 12.5 \mathrm{THz}$. In an extension of this capability, we suggest a novel metrological technique that should allow for absolute comparisons with dense coverage over the region 200-2000 nm.

We begin by considering two-photon excitation of an atomic ladder of the form $1 \rightarrow 2 \rightarrow 3$ with eigenfrequencies $\omega_{12}, \omega_{23}$, and $\omega_{13}=\omega_{12}+\omega_{23}$ by three lasers at frequencies $\omega_{a} \approx \omega_{12}, \omega_{b} \approx \omega_{23}$, and $\omega_{c} \approx 1 / 2 \omega_{13}$ (dotted box in Fig. 1). QI in this system arises from two possible pathways of excitation, the stepwise dipole absorption of two photons at frequencies $\left(\omega_{a}, \omega_{b}\right)$ and the quadrupole two-photon absorption at $\omega_{c}$. Deviations of the excitation frequencies from the triangle equality $\omega_{a}+\omega_{b}=2 \omega_{c}$ result in a modulation of the excited-state population of the form $\rho_{33}(t) \sim$ $\mathcal{A}[1+\mathrm{V} \cos (\Delta \omega t+\phi)]$ at frequency $\Delta \omega=\omega_{a}+\omega_{b}-$ $2 \omega_{c}$ with visibility $\mathrm{V}$ and phase $\phi=\phi_{a}+\phi_{b}-2 \phi_{c}$, where $\phi_{i}$ is the phase of laser $i(i=a, b, c)$. In this scheme the atom acts as a nonlinear mixer that compares $\omega_{a}+\omega_{b}$ with $2 \omega_{c}$; the process corresponds to demodulation of sidebands at frequency offsets $\Delta \nu=$ $\left|\omega_{a}-\omega_{c}\right| / 2 \pi \approx\left|\omega_{c}-\omega_{b}\right| / 2 \pi$ with respect to a local oscillator at $\omega_{c}$. Note that $\rho_{33}(t)$ depends on the excitation scheme and is sinusoidal only in the absence of saturation. Explicit expressions for $\mathcal{A}, \mathrm{V}$, and $\phi$ follow from theoretical analyses based on either perturbative or master equation approaches. ${ }^{4}$

Independently of these more complex treatments, the origin of QI for two-photon excitation can be understood by consideration of Mollow's classic expression, ${ }^{5}$ $\rho_{33} \propto\left\langle\hat{E}^{\dagger} \hat{E}^{\dagger} \hat{E} \hat{E}\right\rangle$, where now $\hat{E}=\hat{E}_{a}+\hat{E}_{b}+\hat{E}_{c}$ is the total excitation field and $\hat{E}_{a, b, c}$ are taken to be of the form $\hat{E}_{i}=\hat{\mathcal{F}}_{i} \exp (-i \Phi \hat{i})$ with $\Phi_{i}=\omega_{i} t+\phi_{i}$. Expanding the fourth-order correlation function of the total field under the assumption of a coherent state for the field $\hat{E}_{c}$ of eigenvalue $E_{c}$, and retaining only those terms that correspond to small two-photon detuning for the transition amplitudes, we find that $\rho_{33} \simeq \alpha_{1}\left|E_{c}\right|^{4}+$ $\alpha_{2}\left\langle\hat{E}_{b}^{\dagger} \hat{E}_{a}^{\dagger} \hat{E}_{a} \hat{E}_{b}\right\rangle+\alpha_{3}\left(E_{c}{ }^{2}\left\langle\hat{E}_{a}^{\dagger} \hat{E}_{b}{ }^{\dagger}\right\rangle+\right.$ c.c. $)$. Note that the first term represents the usual quadrupole twophoton excitation that is proportional to the square of the intensity and the second term corresponds to stepwise dipole absorption at frequencies $\omega_{a}$ and $\omega_{b}$. In the case of nonclassical squeezed fields this second term gives rise to a linear rate of two-photon excitation. ${ }^{6}$ The last term is the focus of the present study and results from interference in two-photon excitation as a function of the overall phase $\Phi=\Phi_{a}+\Phi_{b}-2 \Phi_{c}$ for the fields $\left(\hat{E}_{a}, \hat{E}_{b}, \hat{E}_{c}\right)$.

For an initial proof-of-principle experiment, we excite the $6 S_{1 / 2}, F=4 \rightarrow 6 P_{3 / 2}, F^{\prime}=5 \rightarrow 6 D_{5 / 2}, F^{\prime \prime}=6$ transition $(1 \rightarrow 2 \rightarrow 3)$ in magneto-optically trapped atomic $\mathrm{Cs}$ by three laser beams at wavelengths $\lambda_{a} \approx 852, \lambda_{b} \approx 917$, and $\lambda_{c} \approx 884 \mathrm{~nm}$, as shown in Fig. $1 .^{7}$ The field at $884 \mathrm{~nm}$ originates from a tunable Ti:sapphire laser externally locked to a reference cavity that is in turn locked to the Doppler-free two-photon transition $1 \rightarrow 3$ of $\mathrm{Cs}$ in an auxiliary vapor cell. The field at $852 \mathrm{~nm}$ is provided by a diode laser operated with an external grating and stabilized to the $1 \rightarrow 2$ transition. ${ }^{8}$ Finally, the field at $917 \mathrm{~nm}$ is derived by nonlinear mixing of the 852- and 442-nm (double of 884 ) beams in a parametric amplifier composed of a subthreshold optical parametric oscillator. ${ }^{6}$

The experiment is conducted continuously in two alternating stages that are switched at a rate of $4 \mathrm{kHz}$.

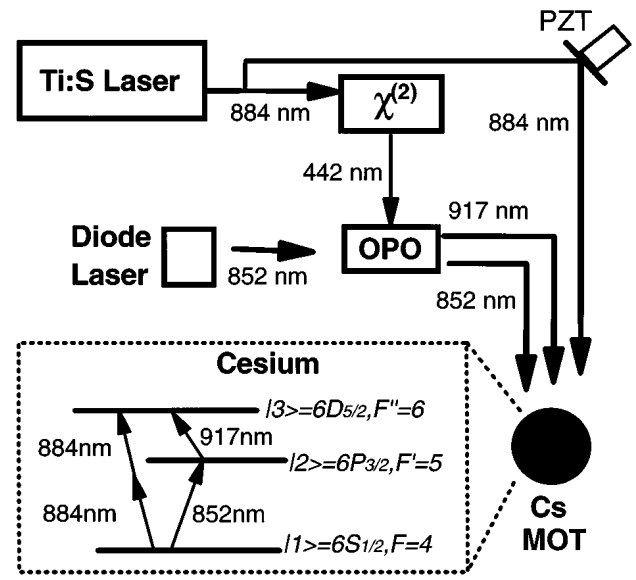

Fig. 1. Experimental setup: PZT, piezoelectric transducer; OPO, optical parametric oscillator; MOT, magnetooptical trap. 
In the first stage atoms are maintained in a MOT. ${ }^{7}$ In the second stage the trapping beams are mechanically chopped off, and the atoms are illuminated with the three lasers at $\lambda_{a, b, c}$ with powers of approximately $0.5 \mathrm{nW}, 0.5 \mathrm{nW}$, and $5 \mathrm{~mW}$, respectively. We monitor the excited-state population $\rho_{33}$ by observing the fluorescent decay $3 \rightarrow 2$ at $917 \mathrm{~nm}$, using an avalanche photodiode.

The phase $\phi$ (and thereby the excited-state population $\rho_{33}$ ) is externally modulated with a sawtooth ramp applied to a moving mirror in the path of the 884-nm laser. During the long linear segment of the ramp, we have $\phi_{c} \rightarrow \phi_{c}(t) \simeq \omega_{m} t$ (with $\omega_{m} / 2 \pi \sim 10-$ $20 \mathrm{~Hz}$ ) and hence $|\phi(t)|=2 \omega_{m} t$. Note that $\Delta \omega=0$ by virtue of the parametric process used to generate $\omega_{b}$ from $\left(\omega_{a}, \omega_{c}\right)$, although the same experiment could be carried out with three totally independent lasers. As illustrated in Fig. 2, a modulation of $\rho_{33}$ at frequency $\Omega_{m}=2 \omega_{m}$ is observed with visibility $\mathrm{V} \simeq 0.3$, thus demonstrating QI in the two-photon excitation process. We stress that the modulation displayed in Fig. 2 corresponds to demodulation of the optical frequency difference $\left|\omega_{a, b}-\left(\omega_{c}+\omega_{m}\right)\right| / 2 \pi \approx 12.5 \mathrm{THz}$.

Because the response of the nonlinear mixer in this particular demonstration is centered around the specific frequencies shown in Fig. 1, its bandwidth is necessarily quite narrow (being intrinsically associated with atomic linewidths), although one can achieve an appreciable response for terahertz detunings for $\left(\omega_{a}, \omega_{b}\right)$ by increasing the incident power to the milliwatt level. However, our scheme of obtaining QI in two-photon excitation is easily generalized to other transitions of the form $1 \rightarrow 2 \rightarrow 3$. Indeed, by expanding our viewpoint to the alkali elements $\mathrm{Li}, \mathrm{Na}, \mathrm{K}, \mathrm{Rb}$, and $\mathrm{Cs}$ but constraining consideration to only ladder transitions for which each of the three wavelengths involved is less than $2 \mu \mathrm{m},{ }^{9}$ we find that there are $\sim 6800$ possible triangles of the form $\omega_{13}=\omega_{12}+\omega_{23}$, each of which can be viewed as a different nonlinear mixer for obtaining QI and hence phase-sensitive detection of $\Delta \omega=\omega_{a}+\omega_{b}-2 \omega_{c}$ for fields of frequencies $\omega_{a} \simeq \omega_{12}, \omega_{b} \simeq \omega_{23}$, and $\omega_{c} \simeq 1 / 2 \omega_{13} \cdot{ }^{10}$ Note that each of these triangles is characterized by its central frequency $1 / 2 \omega_{13}$ and sideband separation $\Delta \nu=$ $1 / 2 \pi\left|\omega_{12}-1 / 2 \omega_{13}\right| \approx 1 /\left.2 \pi\right|^{1 / 2} \omega_{13}-\omega_{23} \mid$. To illustrate the density of coverage, we display in Fig. $3 \Delta \nu$ versus $\lambda_{c}=2 \pi c / \omega_{c}$ for the transitions in the database, with $\Delta \nu$ typically $10-100 \mathrm{THz}$.

The capability for optical demodulation (Fig. 2), together with the numerous possibilities for nonlinear mixing (Fig. 3), suggests that QI can be exploited in two-photon excitation for applications in frequency metrology. In particular, to obtain an absolute measurement of a given target frequency in terms of a handful of reference frequencies, one faces the daunting challenge of bridging intervals that are often comparable to the optical frequency itself. Numerous schemes to conquer these large frequency intervals have been investigated, ${ }^{11-14}$ including resonant multiwave mixing in atomic vapors. ${ }^{15}$

Within this context, we propose a new method for absolute comparison of an (arbitrary) target frequency $\omega_{t}$ one or more reference frequencies $\omega_{r 1}, \omega_{r 2}, \ldots,{ }^{14}$ by ex- ploiting atoms as ultrafast nonlinear mixing elements. As for the actual implementation, we would first construct from the set of available frequencies $\Omega_{0}=$ $\left\{\omega_{t}, \omega_{r 1}, \omega_{r 2}, \ldots\right\}$ a new set $\Omega_{1}=\left\{w_{1}{ }^{\prime}, \omega_{2}{ }^{\prime}, \ldots, \omega_{N_{1}}{ }^{\prime}\right\}$, where one allows for combinations of the form $\omega_{i} \pm \omega_{j}$ with $\left\{\omega_{i}, \omega_{j}\right\} \subseteq \Omega_{0}$. This process can be repeated for as many as $k$ stages to generate a final set of frequencies $\Omega_{k}$, with the number of resulting frequencies growing exponentially in $k$. For example, with two reference and one target lasers, $k=2$ stages of nonlinear conversion produces $\sim 150$ distinct frequencies. Of course, finally, we need only three of these frequencies to mix with the aid of an atom. To decide which three must be generated, we consider all possible triplets $\left(\omega_{a}{ }^{\prime}, \omega_{b}{ }^{\prime} \omega_{c}{ }^{\prime}\right)$ from the set $\Omega=\Omega_{0} \cup \Omega_{1} \ldots \cup \Omega_{k}$ that can be traced back to $\omega_{t}$ and at least one of the $\omega_{\sqrt{i}}$ 's and keep only those

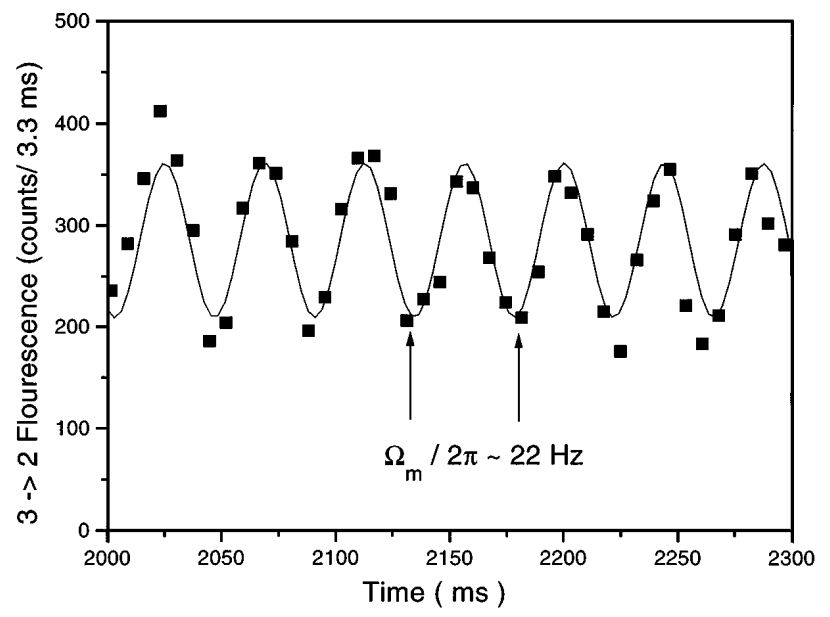

Fig. 2. Fluorescence from the $3 \rightarrow 2$ transition at as a function of time for illumination of the MOT optical trap with three fields at $\lambda_{a, b, c}=(852,917,884) \mathrm{nm}$. Here the relative phase $\phi(t)=2 \phi_{884}(t)-\phi_{852}-\phi_{917}$, where $\phi_{884}(t)=\omega_{m} t$ and $\omega_{m} / 2 \pi=11 \mathrm{~Hz}$. The solid curve is a fit of a constant plus a sinusoidal variation with visibility $\nu \approx 0.3$.

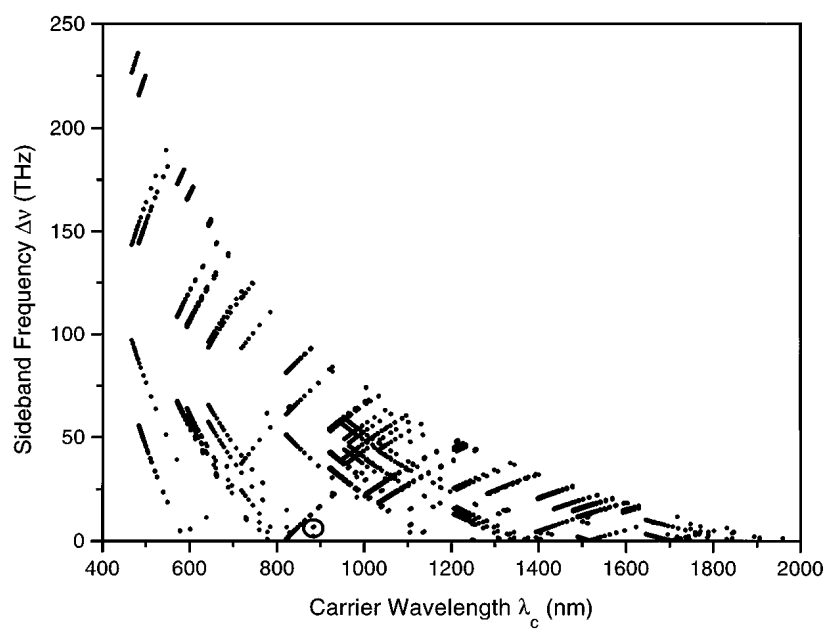

Fig. 3. Nonlinear mixer characteristics for the sidebandto-carrier separation $\Delta \nu$ versus carrier wavelength $\lambda_{c}$ from our database of 6800 transitions in the alkali elements. The circled point corresponds to the current experiment, for which $\lambda_{c}=884 \mathrm{~nm}$ and $\Delta \nu=12.5 \mathrm{THz}$. 
triplets that satisfy $\omega_{a}{ }^{\prime}+\omega_{b}{ }^{\prime}-2 \omega_{c}{ }^{\prime}<\Delta \omega^{\prime}$, where $\Delta \omega^{\prime}$ is the maximum offset that can be either directly detected or compensated by electro-optic modulation $\left(\Delta \omega^{\prime} \leq 500 \mathrm{GHz}\right)$. These triplets are then cross referenced to our database of two-photon transitions with a search algorithm that minimizes the sum $\sum$ of absolute detunings, $\sum=\left|\omega_{a}-\omega_{12}\right|+\left|\omega_{b}-\omega_{23}\right|+\left|\omega_{c}-1 / 2 \omega_{13}\right|$, and thereby chooses the particular atomic transition for the measurement. Alternative criteria can also by considered.

The complexity of possibilities that arise from this database of transitions ${ }^{10}$ and from the set of possible reference wavelengths ${ }^{14}$ does not allow for an explicit algorithm to be presented here. However, a computer program has been developed that analyzes the problem as described above and finds optimized measurement strategies for any target wavelength in the range 200 $2000 \mathrm{~nm}$ for a given set of reference wavelengths. Particular examples of optimized solutions for the measurement of several wavelengths have been found by our program (including the 121.6-nm, Lyman- $\alpha$ transition in atomic hydrogen) and will be discussed elsewhere. $^{16}$

Another possible application of our scheme is in the establishment of frequency standards in the $1.3-$ and $1.5-\mu \mathrm{m}$ bands relevant to optical communications. ${ }^{17}$ Toward this end we have constructed measurement strategies for wavelengths between 1300 and $1500 \mathrm{~nm}$ in increments of $1 \mathrm{~nm} .{ }^{16}$ Yet another potential investigation is a test of the internal consistency of existing frequency standards by employing some as targets and others as references and then making direct comparisons with atoms serving as nonlinear mixers.

Turning finally to the issue of the conversion efficiency for our scheme, we introduce an effective nonlinear coefficient $\mathcal{E}_{\mathrm{NL}}$ for the conversion of the powers $P_{a, b, c}$ into an equivalent power $P_{\mathrm{HET}}^{f}$ for the fluorescence carried at frequency $\delta$, where $2 \omega_{c}=\omega_{a}+\omega_{b}+\delta$. Explicitly, we find $P_{\mathrm{HET}}^{f}=\mathcal{E}_{\mathrm{NL}}\left(P_{c}{ }^{2} P_{a} P_{b}\right)^{1 / 2}$, where $\mathcal{E}_{\mathrm{NL}}=\left(\mathcal{E}_{\mathrm{NL}}^{c} \mathcal{E}_{\mathrm{NL}}^{a b}\right)$, with $\left(\mathcal{E}_{\mathrm{NL}}^{c}, \mathcal{E}_{\mathrm{NL}}^{a b}\right)$ denoting the independent conversion efficiencies from the incident powers $\left(P_{a}, P_{b}, P_{c}\right)$ into fluorescent powers from the $3 \rightarrow 2$ decay, including the collection efficiency $\epsilon$ for a finite solid angle. Note that the form for $P_{\text {HET follows from }}^{f}$ an interference of three field amplitudes equivalent to the $\alpha_{3}$ term in the previous expression for $\rho_{33}$ and that a completely analogous expression can be derived for Hänsch's divide-and-conquer scheme. ${ }^{12}$ Although explicit forms for $\mathcal{E}_{\mathrm{NL}}^{a b, c}$ will be presented elsewhere, ${ }^{4}$ we find for the case of our experiment that $\mathcal{F}_{\mathrm{NL}}^{a b} \sim$ $4 \times 10^{6} / \mathrm{W}$ and $\mathcal{F}_{\mathrm{NL}}^{c} \sim 10^{-8} / \mathrm{W}$, so that the effective nonlinear coefficient $\mathcal{E}_{\mathrm{NL}} \sim 0.2 / \mathrm{W}$ (for a trap density of $10^{9} / \mathrm{cm}^{3}$ and $\epsilon \sim 0.08$ ), which is apparently quite competitive with more conventional mixing schemes even given our (nonoptimal) setup. On the other hand, note that for our initial experiment the saturation powers are $P_{a, b}^{\mathrm{sat}} \sim 0.5 \mathrm{nW}$ and $P_{a, b}^{\mathrm{sat}} \sim 10 \mathrm{~mW}$, so $P_{\mathrm{HET}}^{f} \leq 1 \mathrm{pW}$, which, however, could be significantly increased with a new trap design.
In conclusion, by exploiting QI based on parallel excitation paths for two-photon transitions, we have observed the beat note arising from a small frequency offset $(\approx 10 \mathrm{~Hz})$ between three fields at wavelengths of 852,884 , and $917 \mathrm{~nm}$, demonstrating in this way demodulation of optical offsets of $\pm 12.5 \mathrm{THz}$. By extending the idea of using atoms as nonlinear mixing elements to more general two-photon transitions, we have proposed a scheme for absolute-frequency comparisons between an arbitrary target frequency and a small set of reference frequencies.

This research was supported by the Division of Chemical Science, Office of Basic Energy Science, Office of Energy Research, U.S. Department of Energy. We gratefully acknowledge discussions with J. L. Hall.

*Permanent address, Institute of Physics and Astronomy, Aarhus University, Aarhus C, DK-8000, Denmark.

\section{References}

1. L. Zhu, V. Kleman, X. Li, S. P. Lu, K. Trentelman, and R. J. Gordon, Science 270, 77 (1995).

2. D. W. Schumacher, F. Weihe, H. Q. Muller, and P. H. Bucksbaum, Phys. Rev. Lett. 73, 1344 (1994).

3. M. W. Noel and C. R. Stroud, Phys. Rev. Lett. 75, 1252 (1995).

4. N. Ph. Georgiades, E. S. Polzik, and H. J. Kimble, "Quantum interference in two-photon excitation," Phys. Rev. B (to be published).

5. B. R. Morrow, Phys. Rev. 175, 1555 (1968).

6. N. Ph. Georgiades, E. S. Polzik, K. Edamatsu, H. J. Kimble, and A. S. Parkins, Phys. Rev. Lett. 75, 3426 (1995).

7. N. Ph. Georgiades, E. S. Polzik, and H. J. Kimble, Opt. Lett. 19, 1474 (1994).

8. K. Libbrecht and J. L. Hall, Rev. Sci. Instrum. 64, 2133 (1993).

9. In principle $\mathrm{V}$ and $\Lambda$ two-photon transitions will also exhibit QI. We could likewise include atoms other than the alkalis.

10. Our database of possible triangles was constructed from atomic energy levels for $\mathrm{Li}, \mathrm{Rb}$, and Cs Vols. I-III [C. E. Moore, Atomic Energy Levels, Nat. Bur. Stand. (U.S.) Circ. 467 (National Bureau of Standards, Washington, D.C., 1949)] and from data on the Internet at http:// aeldata.phy.nist.gov/archive/el.html by NIST for Na and $\mathrm{K}$.

11. H. Schnatz, B. Lipphardt, J. Helmcke, F. Riehle, and G. Zinner, Phys. Rev. Lett. 76, 18 (1996).

12. H. R. Telle, D. Meschede, and T. W. Hänsch, Opt. Lett. 15, 532 (1990).

13. D. Lee and N. C. Wong, Opt. Lett. 17, 13 (1992).

14. D. A. Van Baak and L. Hollberg, Opt. Lett. 19, 1586 (1994).

15. V. M. Klementyev, Yu. A. Matyugin, and V. P. Chebotayev, Zh. Eksp. Teor. Fiz. Pis'ma Red. 24, 8 (1976); D. A. Jennings, C. R. Pollock, F. R. Petersen, R. E. Drullinger, K. M. Evenson, J. S. Wells, J. L. Hall, and H. P. Layer, Opt. Lett. 8, 136 (1983).

16. Details will appear on the Internet at http:// www.cco.caltech.edu/ qoptics.

17. D. J. E. Knight, Laser Phys. 4, 345 (1994). 